\title{
Clinical multifactorial analysis of early postoperative seizures in elderly patients following meningioma resection
}

\author{
BO ZHANG ${ }^{1}$, DAN WANG ${ }^{2}$, YUNBAO GUO ${ }^{1}$ and JINLU YU ${ }^{1}$ \\ Departments of ${ }^{1}$ Neurosurgery and ${ }^{2}$ Ophthalmology, First Hospital of Jilin University, Changchun 130021, P.R. China
}

Received October 2, 2014; Accepted January 8, 2015

DOI: $10.3892 /$ mco.2015.493

\begin{abstract}
The aim of the present study was to identify the major factors correlated with early postoperative seizures in elderly patients who had undergone a meningioma resection, and subsequently, to develop a logistic regression equation for assessing the seizures risk. Fourteen factors possibly correlated with early postoperative seizures in a cohort of 209 elderly patients who had undergone meningioma resection, as analyzed by multifactorial stepwise logistic regression. Phenobarbital sodium ( $0.1 \mathrm{~g}$, intramuscularly) was administered to all 209 patients 30 min prior to undergoing surgery. All the patients had no previous history of seizures. The correlation of the 14 clinical factors (gender, tumor site, dyskinesia, peritumoral brain edema (PTBE), tumor diameter, pre- and postoperative prophylaxes, surgery time, tumor adhesion, circumscription, blood supply, intraoperative transfusion, original site of the tumor and dysphasia) was assessed in association with the risk for post-operative seizures. Tumor diameter, postoperative prophylactic antiepileptic drug (PPAD) administration, PTBE and tumor site were entered as risk factors into a mathematical regression model. The odds ratio $(\mathrm{OR})$ of the tumor diameter was $>1$, and PPAD administration showed an OR $>1$, relative to a non-prophylactic group. A logistic regression equation was obtained and the sensitivity, specificity and misdiagnosis rates were 91.4, 74.3 and $25.7 \%$, respectively. Tumor diameter, PPAD administration, PTBE and tumor site were closely correlated with early postoperative seizures; PTBE and PPAD administration were risk and protective factors, respectively.
\end{abstract}

\section{Introduction}

While meningioma is the most common pathological form of intracranial tumor in the elderly (1), such patients

Correspondence to: Dr Jinlu Yu, Department of Neurosurgery, First Hospital of Jilin University, 71 Xinmin, Changchun 130021, P.R. China

E-mail: jinluyu@hotmail.com

Key words: meningioma, elderly, surgical treatment, early seizures, logistic regression analysis often present with multiple complications and have a poor prognosis $(2,3)$. Seizures are the most clinically significant complication $(4,5)$ with early postoperative seizures being defined as those appearing within the first week after surgery (6). Brain edema reaches a maximum level during the first week following surgery, and during this time early postoperative seizures may aggravate brain edema and intracranial hematomas, leading to poor patient prognosis. Therefore, it is important to predict the likelihood of postoperative seizures and subsequently select an optimal prophylactic therapy.

Previous studies have revealed that seizures following meningioma resection are associated with multiple factors. Preoperative seizures, imageable peritumoral brain edema (PTBE) and preoperative dyskinesia are all recognized risk factors for early seizures following meningioma resection, while pre- and postoperative prophylactic antiepileptic drugs appear to protect against such early postoperative seizures. Tumors located in the cortex, near the cortex and near functional areas are more frequently complicated with early postoperative seizures $(7,8)$. However, previous studies have not examined whether these various correlation factors influence each other, whether elderly meningioma patients have distinct pathophysiological backgrounds $(2,3)$ or whether the correlation factors for early postoperative seizures are highly specific. A multifactorial logistic regression analysis was performed of the clinical data obtained from 209 elderly patients who had undergone a meningioma resection for the purpose of further investigating the associations between the occurrence of early seizures following meningioma resection and various clinical correlation factors. An equation was subsequently developed, which used these correlation factors to predict the likelihood of an early post-operative seizure.

\section{Patients and methods}

General information. The clinical data obtained from 209 elderly patients (mean age, 67.5 years; range, 65-76 years) who had been hospitalized and undergone a meningioma resection in the First Hospital of Jilin University (Changchun, China) between December 2000 and December 2010 were reviewed. Of these patients, 35 (17 males and 18 females) had experienced an early postoperative seizure, while 174 patients (58 males and 116 females) had not. All the patients had no history of seizures prior to surgery. 
Table I. Correlation analysis of early postoperative seizures in elderly patients following meningioma resection (1).

\begin{tabular}{lcccccc}
\hline & B & \multicolumn{1}{c}{$\chi^{2}$} & df & P-value & OR & $95 \%$ CI of OR \\
\hline Site (1) & 2.210 & 2.919 & 1 & 0.088 & 9.116 & $(0.722,115.062)$ \\
Site (2) & 0.446 & 0.099 & 1 & 0.753 & 1.562 & $(0.097,25.053)$ \\
PTBE & 2.291 & 15.860 & 1 & $<0.001$ & 9.887 & $(3.201,30.534)$ \\
Diameter (1) & -3.659 & 20.629 & 1 & $<0.001$ & 0.026 & $(0.005,0.125)$ \\
Diameter (2) & -2.434 & 16.476 & 1 & $<0.001$ & 0.088 & $(0.027,0.284)$ \\
Postdrug & -2.892 & 14.719 & 1 & $<0.001$ & 0.055 & $(0.013,0.243)$ \\
Constant & -2.036 & 2.548 & 1 & 0.110 & 0.131 &
\end{tabular}

PTBE, peritumoral brain edema. (1) and (2) refer to the dummy variables in SPSS for logistic regression.

Clinical data collection. The medical records of all the patients were checked for any history of preoperative seizures, aphesis or dyskinesia. All the patients had been diagnosed by head magnetic resonance imaging and/or computed tomography scans, and the gross tumor volume, tumor site and any peritumoral brain edema (PTBE) were preliminarily determined. Phenobarbital sodium ( $0.1 \mathrm{~g}$ intramuscularly) was administered to all 209 patients $30 \mathrm{~min}$ prior to the surgery, and Dilantin $(5 \mathrm{mg} / \mathrm{kg} / \mathrm{day}$, oral) or sodium valproate $(15 \mathrm{mg} / \mathrm{kg} / \mathrm{day}$, oral) was administered to certain patients as a pre- and/or postoperative prophylactic drug. Craniotomy for meningioma resection was performed microscopically for all 209 patients. Tumor adhesion to peritumoral tissues, circumscription between the tumor and normal tissues, blood supply and the original site of the tumor were determined during surgery.

Statistical methods. A logistic regression analysis was performed using SPSS for Windows, Version 15.0 (SPSS, Inc., Chicago, IL, USA). Correlations of all the variables with early postoperative seizures were determined by multifactorial logistic regression analysis. Variables were selected by stepwise regression analysis. The influence of each factor on early postoperative seizures was analyzed by fitting a logistic regression equation and a multifactorial logistic regression equation was established. $\mathrm{P}<0.05$ was considered to indicate a statistically significant difference.

\section{Results}

Main factors possibly associated with early postoperative seizures. A total of 14 factors (gender, tumor site, dyskinesia, PTBE, tumor diameter, pre- and postoperative prophylaxes, surgery time, tumor adhesion, circumscription, blood supply, intraoperative transfusion, original site of the tumor and dysphasia) were grouped for analysis. The tumor site groups were further divided into three subgroups: i) Superficial supratentorial subgroup: Frontal, frontotemporal, frontoparietal, temporal, temporoparietal, temporo-occipital, parietal, parietooccipital and occipital regions; ii) deep supratentorial subgroup: Sphenoid ridge, middle and anterior cranial fossae and saddle area; and iii) subtentorial subgroups, including cerebellar hemispheres, petroclival and cerebellopontine angle. The tumor diameter, surgery time and blood supply groups were each individually divided into three subgroups: Tumor diameter $1(<3 \mathrm{~cm}), 2(3-5 \mathrm{~cm})$ and $3(>5 \mathrm{~cm})$; surgery time subgroups $1(<2 \mathrm{~h}), 2(2-4 \mathrm{~h})$ and $3(>4 \mathrm{~h})$; and blood supply subgroups 1 (rich), 2 (medium) and 3 (poor). Original tumor site groups were subdivided into five subgroups: i) Supratentorial convexity; ii) falx: Cerebral falx and sagittal sinus; iii) deep basicranial and intracranial: Middle cranial fossa, optic nerve, brainstem, petroclivas, sphenoid ridge, anterior cranial fossa, choroid plexus, tuberculum sellae and olfactory sulcus; iv) tentorium cerebelli: Tentorium cerebelli; and v) subtentorial convexity. The remaining factors were established as binary variables.

The logistic regression model was fitted using the regrouped factors, and model variables were selected by stepwise regression analysis. The four correlation factors: Tumor diameter (Diameter), postoperative prophylactic antiepileptic drug administration (Postdrug), PTBE and tumor site (Site), were entered into the logistic regression model, and the other factors were excluded. The odds ratio (OR) of the tumor diameter was $>1$, making it a risk factor, while the OR of PPAD (postdrug) was $<1$, making it a protective factor $(\mathrm{P}<0.05)$ (Table I). Two independent variables ('Site' and 'Diameter') were dummy-coded (Table II).

Statistical analysis. Statistical calculations indicated that four factors (Diameter, Postdrug, PTBE and Site) were significantly correlated with early postoperative seizures in the elderly patients $(\mathrm{P}<0.05)$ (Tables I and II).

Based on these results, the possible occurrence of an early postoperative seizure could be represented by the following logistic regression equation:

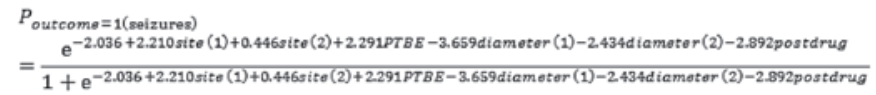

$\left(\chi^{2}\right.$ goodness of fit test for the logistic regression test: $\mathrm{P}=0.713$, sensitivity $=74.3 \%$, specificity $=91.4 \%$ ).

The $\chi^{2}$ goodness of fit test was statistically significant as the $\mathrm{P}$-value was $>0.05$. Application of this regression equation to data from each patient produced a number ranging from zero to one. A value of 1 or close to 1 indicated a high probability of an early postoperative seizure, while a value close to zero indicated a low probability of an early postoperative seizure. 
Table II. Correlation analysis of early postoperative seizures in elderly patients following meningioma resection (2).

Parameter coding

\begin{tabular}{lcccccc}
\cline { 4 - 6 } Group & Team & Frequency & $(1)$ & (2) & (3) & (4) \\
\hline Diameter & 1.00 & 71 & 1.000 & 0.000 & & \\
& 2.00 & 95 & 0.000 & 1.000 & & \\
\multirow{3}{*}{ Site } & 3.00 & 43 & 0.000 & 0.000 & & \\
& 1.00 & 136 & 1.000 & 0.000 & & \\
& 2.00 & 51 & 0.000 & 1.000 & & \\
& 3.00 & 22 & 0.000 & 0.000 & & \\
\hline
\end{tabular}

(1)-(4) refer to the dummy variables in SPSS for logistic regression.

\section{Discussion}

Meningioma is the most common pathological form of intracranial tumor in the elderly (1). Multiple factors lead to an unfavorable prognosis and markedly higher incidence of complications in elderly patients following surgery for intracranial meningioma (9-11). Elderly patients may have various degrees of compromised physical function and complications with comorbidities in other systems. In addition, meningiomas in the elderly are characterized by their growth and produce only slightly increased intracranial pressure and incidences of atypical clinical manifestations due to brain atrophy. Additionally, meningioma tumors are usually located in areas of the brain not associated with physical functions $(12,13)$.

Seizures following intracranial meningioma resection represent a serious complication, and the molecular and cellular mechanisms of tumor-induced seizures are not fully understood. However, previously suggested mechanisms for seizure include focal cortical hypoxia, a direct mass effect, PTBE, peripheral cortical damage and changes in levels of excitatory amino acids. Seizures tend to be the first symptom of an intracranial tumor and may further aggravate brain damage and increase the risk of an unfavorable prognosis based on the underlying disease $(4,14)$. In particular, early postoperative seizures occur within one week following surgery and often aggravate the pathological damage to brain tissue and biochemical changes in nerves caused by underlying disease (15). Information gained from a study of clinical factors correlated with early postoperative seizures in elderly patients following meningioma resection may significantly help to improve patient prognosis. Previous studies have reported that seizures following meningioma resection are associated with multiple clinical factors $(4,5,7,8)$, such as a history of preoperative seizures, imageable PTBE and preoperative dyskinesia. Tumors located in the cortex, near cortex and near functional areas are more likely to be associated with postoperative seizures, while pre- and postoperative prophylactic antiepileptic drugs have been shown to reduce the incidence of seizures. However, no previous study has investigated the clinical factors associated with early postoperative seizures in elderly patients following meningioma resection. The present study used multiple regression analysis to examine the correlation of early postoperative seizures with several clinical factors (gender, tumor site, dyskinesia, PTBE, tumor diameter, pre- and postoperative prophylaxes, surgery time, tumor adhesion, circumscription, blood supply, intraoperative transfusion, original site of the tumor and dysphasia) in a cohort of 209 elderly patients who had undergone a meningioma resection.

Meningiomas are usually slow growing tumors, and their induced seizures are often a result of mechanical compression. Compression of peritumoral brain tissue may destroy the neural network structure; however, neuronal shortcuts leading to a histological basis for interictal epileptiform discharges may appear following pathological reconstruction of the network $(16,17)$. Intracalvarial invasion by a meningioma produces mechanical compression and localized damage to the brain, and the incidence of seizures increases with the volume occupied. In addition, slow growing tumors may cause edema and ischemia in surrounding brain tissues, secondary brain parenchymal atrophy and gliosis, neuronal metabolic disturbance and abnormal depolarization of cell membranes. Such changes lead to epileptic seizures evoked by abnormal neuronal discharges during tumor growth $(14,18)$. The majority of the elderly patients in the present study cohort showed signs of pathological underlying brain parenchymal atrophy and cerebral ischemia. The slow growth of their meningiomas and subsequent development of clinical manifestations may have resulted in chronically and mechanically compressed peritumoral brain tissues and damaged neural network structures, which provided a histological basis for seizures. In the study, a multifactorial logistic regression analysis was performed of the clinical data obtained from 209 elderly patients who had undergone a meningioma resection. The results showed that the OR was maximal in tumor diameter group 3 (1.000) and minimal in tumor diameter group $1(0.026)$, suggesting that the odds of an early postoperative seizure increased with increasing tumor diameter.

Results of a retrospective study by Kawugachi et al (19) indicated that PTBE was a significant epileptogenic factor. Tumor-evoked compression or stimulation of brain tissue results in gliosis, which later causes PTBE, tumidness, insufficient blood supply, anoxia, gradual brain atrophy, cerebral sclerosis, proliferation of glial cells and fibers, loss of partial ganglion cells and the formation of fine insular phagocyte-like lesions. The membranes of these altered pathological nerve cells can stimulate allergic reactions and cause clinical seizures by sudden and transient neuronal discharges induced by endogenous or exogenous stimuli (16). According to Lieu and Howng (5), clinically significant seizures can be caused by supratentorial or convexity meningiomas complicated with severe PTBE. The present study concluded that the OR of PTBE was 9.887, suggesting that the odds of an early postoperative seizure occurring in an elderly patient with meningioma complicated with definite PTBE were almost 10-fold greater than those in a patient without PTBE. This result is in accordance with the above-mentioned study. Previous studies (20-22) have demonstrated that perioperative administration of prophylactic antiepileptic drugs can prevent partial seizures from evolving into persistent ones, and markedly reduce or avoid seizure-induced brain damage. Other studies (23-25) have indicated that prophylactic Dilantin and 
sodium valproate can reduce the incidence of postoperative seizures, and that no significant difference was noted between the results achieved with the two drugs. Therefore, the present study did not distinguish between these two prophylactic antiepileptic drugs when they were used in patient treatment. The results showed a significant difference in the incidence of postoperative seizures in elderly patients who did and did not receive a prophylactic antiepileptic drug. However, use of preoperative prophylactic antiepileptic drugs was not included in the multifactorial stepwise logistic regression; therefore, no statistical evidence is available to indicate that the results were different from those reported in previous studies conducted with patients of all ages (15). In this study, phenobarbital sodium, a temporary prophylactic antiepileptic drug, was administered intramuscularly to all patients $30 \mathrm{~min}$ before surgery, and this factor may have impacted the correlation between preoperative administration of prophylactic antiepileptic drugs and early postoperative seizures. However, the elderly patients in the present study were relatively sensitive to antiepileptic drugs, and any impact should have been evident. Furthermore, empiric therapy may have also influenced the present results, thus results of the $\chi^{2}$ test were not significantly correlated with early seizures prior to meningioma resection. Further confirmation of the effects of antiepileptic drugs is also required as the plasma concentrations of these agents were not determined following pre- and postoperative administration in the study. However, it is reasonable to assume that administration of an antiepileptic drug during the perioperative period may significantly reduce morbidity from early postoperative seizures.

The site of a meningioma may be a decisive factor affecting its likelihood to induce epileptic seizures. Previous studies $(6,26)$ suggested that supratentorial and cerebellar convexity meningiomas are significant causes of seizures. Tumors in areas of the brain associated with reasoning, emotions and motor activities are known to more easily cause epileptic seizures, and tumors in or near the cortex are associated with a higher incidence of seizures compared to tumors in other locations. Pathological changes of deep tissue occur less frequently. Regardless of patient history of preoperative seizures, parasagittal meningiomas located in the front third of the superior sagittal sinus are associated with a high incidence of postoperative seizures due to their close proximity to the central region of the brain. Penfield et al (27) reported that the incidence of seizures caused by intracranial tumors in the supratentorial, saddle and subtentorial areas were 50.0, 5.7 and $2.5 \%$, respectively. These findings may be associated with whether a balance between excitation and inhibition is more easily destroyed in different areas of the brain. The results of the present study support the above findings. Compared to the subtentorial group, the ORs of the superficial and deep supratentorial groups were 9.116 and 1.562 , respectively. Therefore, the superficial and deep supratentorial tumor groups were at a higher risk for seizure compared to the subtentorial group, and the superficial supratentorial group was at the highest risk for early postoperative seizures.

In summary, a multifactorial stepwise logistic regression analysis of 14 clinical factors that may be correlated to early postoperative seizures in elderly patients who had undergone resection for meningioma was performed. Following exclusion of the interference factors, four clinical factors (tumor diameter, administration of a postoperative prophylactic antiepileptic drug, PTBE and tumor site) were entered into a regression model as independent factors correlated with seizures. Furthermore, a stepwise logistic regression equation with a statistically significant goodness of fit test ( $P>0.05)$ was obtained. This equation can be used to objectively predict disease prognosis and provide guidance for postsurgical treatment, while laying a foundation for further systematic studies.

\section{References}

1. Kuratsu $J$ and Ushio Y: Epidemiological study of primary intracranial tumours in elderly people. J Neurol Neurosurg Psychiatry 63: 116-118, 1997.

2. Patil CG, Veeravagu A, Lad SP and Boakye M: Craniotomy for resection of meningioma in the elderly: a multicentre, prospective analysis from the national surgical quality improvement program. J Neurol Neurosurg Psychiatry 81: 502-505, 2010.

3. Poon MT, Fung LH, Pu JK and Leung GK: Outcome comparison between younger and older patients undergoing intracranial meningioma resections. J Neurooncol 114: 219-227, 2013.

4. González-Martínez JA and Najm IM: Meningiomas and epilepsy. meningiomas: Springer: 243-246, 2009.

5. Lieu AS and Howng SL: Intracranial meningiomas and epilepsy: incidence, prognosis and influencing factors. Epilepsy Res 38: 45-52, 2000.

6. Mauro AM, Bomprezzi C, Morresi S, et al: Prevention of early postoperative seizures in patients with primary brain tumors: preliminary experience with oxcarbazepine. J Neurooncol 81: 279-285, 2007.

7. Marosi C, Hassler M, Roessler K, et al: Meningioma. Crit Rev Oncol Hematol 67: 153-171, 2008.

8. Chow SY, Hsi MS, Tang LM and Fong VH: Epilepsy and intracranial meningiomas. Zhonghua Yi Xue Za Zhi (Taipei) 55: 151, 1995.

9. Markides KS, Black SA, Ostir GV, Angel RJ, Guralnik JM and Lichtenstein M: Lower body function and mortality in Mexican American elderly people. J Biol Sci Med Sci 56: M243-M247, 2001.

10. Bernstein M: Brain tumour surgery in the elderly: a brief reappraisal. Can J Surg 39: 147-150, 1996.

11. Caroli M, Locatelli M, Prada F, et al: Surgery for intracranial meningiomas in the elderly: a clinical-radiological grading system as a predictor of outcome. J Neurosurg 102: 290-294, 2005.

12. Fang C, Tan Y, Cui Z, Shi Y, Wang J and Di H: Clinical and pathological features in 49 elderly patients with meningiomas. Neural Regen Res 2: 574-576, 2007.

13. Wofford JL, Moran WP, Wilson TA and Velez R: Clinical presentation of meningioma in the elderly. J Am Geriatr Soc 41: $122,1993$.

14. Wyllie E, Comair YG, Kotagal P, Bulacio J, Bingaman W and Ruggieri P: Seizure outcome after epilepsy surgery in children and adolescents. Ann Neurol 44: 740-748, 1998.

15. Zhang B, Zhao G, Yang HF, Wang D, Yu JL and Huang HY: Assessment of risk factors for early seizures following surgery for meningiomas using logistic regression analysis. J Int Med Res 39: 1728-1735, 2011.

16. Beaumont A and Whittle IR: The pathogenesis of tumour associated epilepsy. Acta Neurochirurgica (Wien) 142: 1-15, 2000.

17. Shamji MF, Fric-Shamji EC and Benoit BG: Brain tumors and epilepsy: pathophysiology of peritumoral changes. Neurosurg Rev 32: 275-284, 2009.

18. van Breemen MS, Wilms EB and Vecht CJ: Epilepsy in patients with brain tumours: epidemiology, mechanisms and management. Lancet Neurol 6: 421-430, 2007.

19. Kawaguchi T, Kameyama S and Tanaka R: Peritumoral edema and seizure in patients with cerebral convexity and parasagittal meningiomas. Neurol Med Chir (Tokyo) 36: 568-574, 1996.

20. De Santis A, Villani R, Sinisi M, Stocchetti N and Perucca E: Add-on phenytoin fails to prevent early seizures after surgery for supratentorial brain tumors: a randomized controlled study. Epilepsia 43: 175-182, 2002. 
21. Lee ST, Lui TN, Chang CN, et al: Prophylactic anticonvulsants for prevention of immediate and early postcraniotomy seizures. Surg Neurol 31: 361-364, 1989

22. Zachenhofer I, Donat M, Oberndorfer S and Roessler K: Perioperative levetiracetam for prevention of seizures in supratentorial brain tumor surgery. J Neurooncol 101: 101-106, 2011.

23. Foy PM, Chadwick DW, Rajgopalan N, Johnson AL and Shaw MD: Do prophylactic anticonvulsant drugs alter the pattern of seizures after craniotomy? J Neurol Neurosurg Psychiatry 55: 753-757, 1992

24. Franceschetti S, Binelli S, Casazza M, et al: Influence of surgery and antiepileptic drugs on seizures symptomatic of cerebral tumours. Acta Neurochir (Wien) 103: 47-51, 1990.
25. Temkin NR: Antiepileptogenesis and seizure prevention trials with antiepileptic drugs: meta. analysis of controlled trials. Epilepsia 42: 515-524, 2001.

26. O'Connell J: Parasagittal and falx meningiomas. Proc Royal Soc Med 64: 584, 1971.

27. Penfield W, Erickson T and Tarlov I: Relation of intracranial tumors and symptomatic epilepsy. Arch Neurol Psychiatry 44: 300, 1940. 